\title{
Peptides Targeting Fms-Related Tyrosine Kinase-4 Activate the Function of Natural Killer Cells in Acute Myeloid Leukemia
}

\author{
Soojin Park ${ }^{1,2}$, Hee-Je Kim ${ }^{2,3}$, Hee-Sun Hwang ${ }^{2}$, A-Reum Han ${ }^{4}$ \\ ${ }^{1}$ Department of Biomedicine E Health Sciences, College of Medicine, The Catholic University of Korea, Seoul, Korea \\ ${ }^{2}$ Lenkemia Research Institute, College of Medicine, The Catholic University of Korea, Seoul, Korea \\ ${ }^{3}$ Catholic Hematology Hospital, Seoul St. Mary's Hospital, College of Medicine, The Catholic University of Korea, Seoul, Korea \\ ${ }^{4}$ Department of Biomedical Science, CHA University, Seongnam, Korea
}

Background and Objectives: The increased expression for the Fms-related tyrosine kinase-4 (FLT-4, known as VEGFR-3) is relevant to dysfunctional natural killer (NK) cells in acute myeloid leukemia (AML). MAZ51 (M), a VEGFR-3 inhibiting chemical, was effectively restored the function of NK cells via the high expression of interferon-gamma (IFN- $\gamma$ ) in NK cells, as shown in our previous study. Although tremendous amount of clinical data using peptides are currently available in real clinic, peptides targeting FLT-4 in modulating immune cells such as NK cells are not fully elucidated.

Methods and Results: In present study, we developed peptides targeting FLT-4 (P), which is inhibiting an affinity for AML-NK expressing FLT-4 in vitro and in vivo. Bone marrow (BM) and peripheral blood (PB) mononuclear cells (MNCs) from AML patients were treated with combinational cocktails of the three agents including $\mathrm{P}, \mathrm{M}$, ara-C (A) and FLT-4 expression and IFN- $\gamma$ release were examined. In an AML mouse model, IFN- $\gamma$ expression were examined in T and NK cells from mouse BM, spleen, and liver to address relevance between peptides and immune cell activation. We found that AML-NK cells both in human and mouse samples showed a gradual increase the IFN- $\gamma$ levels compared to the controls. There was a trend toward a reduction in leukemic blasts in the BM, spleen, and liver from the AML mice, when we compared the effects of combinational treatments.

Conclusions: Our results suggest that the function of AML-NK cells was synergistically activated by $\mathrm{P}$ in combination with $M$ or $\mathrm{A}$.

Keywords: FLT-4, Peptide, AML, Interferon- $\gamma$, Natural killer cell

Received: April 30, 2021, Revised: May 10, 2021,

Accepted: May 12, 2021, Published online: June 30, 2021

Correspondence to Hee-Je Kim

Leukemia Research Institute, College of Medicine, The Catholic University of Korea, 222 Banpo-daero, Seocho-gu, Seoul 06591, Korea; Catholic Hematology Hospital, Seoul St. Mary's Hospital, College of Medicine, The Catholic University of Korea, 222 Banpo-daero, Seocho-gu, Seoul 06591, Korea

Tel: +82-2-2258-6054, Fax: +82-2-599-3589

E-mail: cumckim@catholic.ac.kr

(a) This is an open-access article distributed under the terms of the Creative Commons Attribution Non-Commercial License (http://creativecommons.org/ licenses/by-nc/4.0/), which permits unrestricted non-commercial use, distribution, and reproduction in any medium, provided the original work is properly cited.

Copyright (c) 2021 by the Korean Society for Stem Cell Research

\section{Introduction}

Vascular neonatal factors, such as VEGF ligands and VEGF receptors, have previously been found to play important pathogenic and prognostic roles as markers for a variety of human tumors including AML and to be promising anti-cancer treatment targets (1). Many aspects of clinically meaningful features derived from VEGF-C and FLT-4-related studies indicated the high rate of growth and survival of leukemia cells (2). These features were closely related to the invasive infiltration of leukemic cells through the lymphatics and blood vessels of patients with AML (3). Meanwhile, AML patients have a relatively small 
number of NK cells, which are relatively low functioning, with decreased interferon (IFN)- $\gamma$ expression $(4,5)$. AML-NK cells represent unique lymphocyte subgroups separate from $\mathrm{T}$ cells and $\mathrm{B}$ cells (6). Although NK cells have a key immunologic role as an innate immune system component even in patients with AML, they are less functional or even functionless compared to those in healthy people ( 7 , 8). FLT-4 is highly expressed on NK cells in AML patients, suggesting that low functioning AML-NK cells may be associated with higher levels of FLT-4, while NK cells in AML patients have reversed their functional aspects, with lower expressions of IFN- $\gamma(8,9)$. The findings of FLT-4 antagonist treatment, indicating the probability of a new therapeutic tool in AML-NK immunotherapy or similar approaches, are promising in real clinic use. Likewise, our previous studies underlined the importance of developing some clinical-grade peptides for low-functioning AML-NK cells with VEGF-C (VEGF-C)/FLT-4dependent or related systems, mechanisms, or pathophysiologic backgrounds (10). Peptides can be utilized directly as a cytotoxic agent through various mechanisms or can act as a carrier of cytotoxic agents and radioisotopes by specifically targeting cancer cells. Peptide-based combinational therapy is emerging as an important strategy to overcome cancer $(11,12)$. While large proteins including monoclonal antibody have some drawbacks to be solved such as delivery difficulty into tumor and non-specific uptake into the reticuloendothelial system, peptides contain many advantages including small size, easy synthesis, and accessibility for tumor $(13,14)$. Above all, the biggest benefit to peptides is that it can be directly applied in clinical trials with diverse combination regimens (15). Based on our previous data, we developed the novel peptide that can target FLT-4 to inhibit its activity on AML-NK cells.

Many novel chemotherapeutic drugs for AML have been introduced and applied with diverse combinational treatments including peptides. However, no study reported for peptide targeting FLT-4 which helps to reduce blasts by increased IFN- $\gamma$ expression of AML-NK cells (16). In present study, we investigated whether FLT-4 inhibition and NK cell activation achieved by using a newly designed FLT-4 inhibitory peptide in vitro and in vivo were achievable and useful goals. This study suggested that the dysfunctional AML-NK cells were synergistically restored by peptide, when $M$ and A were treated together.

\section{Materials and Methods}

\section{Murine cell line}

The murine myeloid leukemia cell line C1498 (a murine
AML cell line, TIB-49; ATCC, Manassas, VA, USA) was used. C1498 cells were cultured in Dulbecco's Modified Eagle's Medium (DMEM; Gibco, Grand Island, NY, USA), 10\% fetal bovine serum (FBS; Gibco), and penicillin-streptomycin (Pen-Strep; Gibco) solution in a humidified atmosphere of $5 \%$ at $37^{\circ} \mathrm{C}$.

\section{Peptide}

The FLT-4 peptide ligand used in this experiment inhibits FLT-4 protein activity and its sequence was listed in Table 1. Peptide was designed by randomly cutting peptides with sizes not exceeding 15 -mers based on sequences located in Thr446 and Lys516 of the D5 region bound by strong disulfide bonds in the FLT-4 Fc region (Peptron, Daejeon, Korea). In the experiments, $20 \mathrm{mg} / \mathrm{kg}$ in vivo and $7.5 \mu \mathrm{M}$ in vitro were used. The FLT-4 peptide (termed P) was dissolved in distilled water (D.W.).

\section{Leukemic mouse model}

C57BL/6J mice were purchased from the Jackson Laboratory (Bar Harbor, ME, USA). Researchers at the Catholic University of Korea followed ethical guidelines to manage the mice in a pathogen-free environment. A total of 12 female mice were used in this experiment, and their initial body weight (5 weeks) was 17 18 g. At the time of treatment (7 weeks old), it was 19 20 g. All protocols for testing the animals were approved by the Catholic University of Korea's Institutional Animal Care and Use Committee (CUMC-2019-0135-01). For the syngeneic model, $2 \times 10^{6}$ C1498 cells suspended in $200 \mu 1$ of phosphate-buffered saline (PBS) were intravenously injected into 7-week-old mice. The treatment began seven days after the injection. As described in Fig. 1, peripheral blood (PB) was collected from the facial vein and tissue samples from the organs were collected from the euthanized mice on day 18 post-injection. The collected PB and tissues were analyzed by fluorescent-activated cell sorter (FACSCanto II; BD Bioscience, Santa Clara, CA, USA) analysis. MAZ51 (termed M; 16168; Cayman chemical, Michigan, USA) was injected intraperitoneally at $8 \mathrm{mg} / \mathrm{kg}$ (200 $\mu 1$ in $1 \%$ carboxymethylcellulose calcium (Sigma, St. Louis, MO, USA), 0.2\% Tween 80 (P1754; Sigma)) daily for three days. The $\mathrm{P}$ was injected intravenously daily for six days at $20 \mathrm{mg} / \mathrm{kg}$ dissolved in $200 \mu 1$ of PBS. Cytosine

Table 1. Peptide sequence

\begin{tabular}{cc}
\hline & Sequence \\
\hline Peptide & RQALTCTAYGVP \\
\hline
\end{tabular}




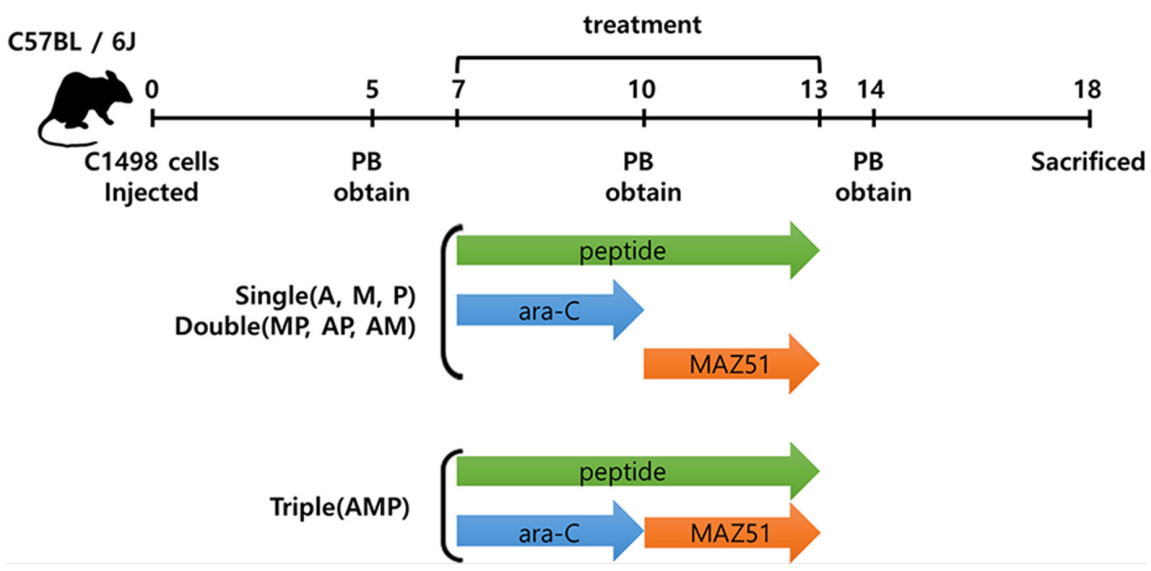

Fig. 1. In vivo experiment schedule. Schematic diagram of in vivo experiment. P: peptide, M: MAZ51, A: ara-C.

$\beta$-D-arabinofuranoside (ara-C; termed A; Sigma, C1768) was injected intraperitoneally daily for three days at 100 $\mathrm{mg} / \mathrm{kg}$ in $200 \mu \mathrm{l}$ of PBS.

\section{Human samples}

All experiments were conducted with the approval of the Catholic Human Ethics Review Committee (KC19TESI0462). Bone marrow (BM) and $\mathrm{PB}$ samples were collected from 23 patients with primary AML according to the AML subtype classification designated by the World Health Organization (WHO). BM and PB mononuclear cells (MNCs) were fractionated by density gradient centrifugation using Ficoll-Paque ${ }^{\circledR}$ Plus (17-1440-03; GE Healthcare Life Sciences, Piscataway, NJ, USA). The clinical characteristics and experimental information for AML patients enrolled in this study are listed in Table 2. The collected $\mathrm{BM}$ and $\mathrm{PB}$ MNCs were grown in a humidified incubator of $5 \% \mathrm{CO}_{2}$ at $37^{\circ} \mathrm{C}$ and cultured in RPMI 1640 medium (Gibco) supplemented with 10\% FBS (Gibco) and Pen-Strep antibiotics (Gibco) for 24 hours for each monotherapy, double (MAZ51 (M) and peptide (P) dual-treated group; termed MP group, A and peptide (P) dual-treated group; termed AP group, A and $M$ dual-treated group; termed AM group) and triple combination therapies of $M$ (Cayman chemical, $7.5 \mu \mathrm{M}$ in stock in DMSO), $\mathrm{P}$ (Peptron, $7.5 \mu \mathrm{M}$ stock in D.W.), and A (Sigma, $10 \mu \mathrm{M}$ stock in D.W.). All experiments were performed in single, dual, and triple combinations.

\section{Quantitative reverse transcription-polymerase chain reaction}

Total RNA was isolated using TRIzol reagent (15596018; Invitrogen), and cDNA synthesis was performed using the Transcriptor First-Strand cDNA Synthesis Kit (04 897030 001; Roche, Bacel, Switzerland). qRT-PCR was performed with LightCycler ${ }^{\circledR} 480$ SYBR Green I Master Mix (04 707
516 001; Roche, Bacel, Switzerland). The TaqMan primers (Biosearch Technologies, Novato, CA, USA) used in this study were mouse GAPDH (Forward - CGTGTTCCTACC CCCAATGT, Reverse - TGTCATCATACTTGGCAGGTT T), mouse LAMP 1 (Forward - GTCTTCTTCGTGCCGG CGT, Reverse - GCAGGTCAAAGGTCATGTTCTT), mouse Perforin (Forward - GACTGCTGCCCACGACAGA, Reverse TGCCCGGAAATTGCTTACC), mouse IFN- $\gamma$ (Forward GTCCAGCGCCAAGCATTC, Reverse - GCTGGATTCCG GCAACAG), and mouse granzyme B (Forward - CCCAG GCGCAATGTCAAT, Reverse - CCCCAACCAGCCACAT AGC). The target gene mRNA expression was calculated using a comparative CT method. All target gene expressions were normalized to GAPDH expression in multiplexed reactions performed twice. The difference in CT values for each target mRNA was calculated by subtracting the mean value of GAPDH expression (relative expression $=2-\Delta \mathrm{CT}$ ).

\section{Flow cytometry}

The collected BM MNCs and PB MNCs were incubated together in $100 \mu \mathrm{l}$ PBS. The human antibodies APC-labelled mouse anti-human CD56 (555518; BD Biosciences), FITC-labelled mouse anti-human CD3 (555339; BD Biosciences), and PE-labelled anti-human VEGFR-3 (FLT-4) antibody (356204; BioLegend, San Diego, CA, USA), APC Mouse IgG1, $\kappa$ Isotype Control (555751; BD Biosciences), FITC Mouse IgG2a, $\kappa$ Isotype Control (555573; BD Biosciences), PE Mouse IgGl, $\kappa$ Isotype Ctrl (FC) Antibody (400114; BioLegend), PE Mouse IgG1, $\kappa$ Isotype Control (559320; BD Biosciences) were used. The collection and analysis, and the analysis of intracellular IFN- $\gamma$ expression were performed as described above. In vivo model, PB, spleen, and liver cells were obtained from the AML mice on day 18 post-injection of C1498. The BM cells were flushed from the mouse femurs, suspended in 100 
Table 2. Clinical characteristics and experimental information for AML patients

\begin{tabular}{|c|c|c|c|c|c|c|}
\hline Patient & Fig. $2 B$ & Fig. 2C & Age & Sex & WBC count $\left[10^{9} / l\right]$ & Cytogenetic anomalies \\
\hline 1 & BM & & 21 & $\mathrm{~F}$ & 4.45 & $46, X X, t(8 ; 21)(q 22 ; q 22)[20]$ \\
\hline 2 & BM & & 68 & $\mathrm{~F}$ & 14.29 & $46, X X, i(14)(q 10)[17] / 49$, idem, $+8+9+21[3]$ \\
\hline 3 & BM & & 67 & $\mathrm{~F}$ & 3.39 & $46, X X[20]$ \\
\hline 4 & BM & & 75 & $\mathrm{~F}$ & 1.75 & $47, X X,+8[20]$ \\
\hline 5 & BM & & 59 & M & 158.98 & $46, X Y[20]$ \\
\hline 6 & BM & PB & 44 & M & 233.51 & $46, X Y, \operatorname{dup}(1)(\mathrm{p} 22 \mathrm{p} 34.3), \operatorname{del}(5)(\mathrm{q} 31), \mathrm{t}(10 ; 11)(\mathrm{q} 22 ; \mathrm{q} 23)[18] / 46, \mathrm{XY}[2]$ \\
\hline 7 & BM & & 55 & $\mathrm{~F}$ & 6.46 & $45, X,-X, t(8 ; 21)(q 22 ; q 22)[20]$ \\
\hline 8 & BM & & 45 & $\mathrm{~F}$ & 57.37 & $46, X X, t(8 ; 21)(q 22 ; q 22)[8] / 45$, idem,-X[17]/46,XX[5] \\
\hline 9 & BM & & 38 & $\mathrm{~F}$ & 11.22 & $46, X X[20]$ \\
\hline 10 & BM & & 71 & $\mathrm{~F}$ & 2.68 & $46, X X[20]$ \\
\hline 11 & BM & & 55 & M & 143.31 & $47, X Y,+8[20]$ \\
\hline 12 & BM & & 51 & $\mathrm{~F}$ & 3.57 & $46, X X[20]$ \\
\hline 13 & BM & & 85 & M & 22.67 & $\begin{array}{l}\text { 46,XY, add(4)(p15),-16[9]/45,idem, add (11)(q23) }[4] / 45, Y, t(X ; 4 ; 3) \\
(p 22.1 ; q 25 ; p 21)[5] / 46, X Y[2]\end{array}$ \\
\hline 14 & & PB & 43 & M & 103.41 & $\begin{array}{l}46, X Y,+1+\operatorname{der}(1 ; 22)(q 10 ; q 10)[3] / 46, \text { idem,t(6;12)(p21.1;p13) } \\
{[16] / 46, X Y[1]}\end{array}$ \\
\hline 15 & & PB & 33 & $\mathrm{~F}$ & 9.28 & $46, X X[20]$ \\
\hline 16 & & PB & 30 & $\mathrm{~F}$ & 310.5 & $46, X X[20]$ \\
\hline 17 & & PB & 57 & $M$ & 274.08 & $46, X Y[8]$ \\
\hline 18 & & PB & 40 & $\mathrm{~F}$ & 132.48 & $46, X X[20]$ \\
\hline 19 & & PB & 68 & M & 148.24 & $46, X Y[20]$ \\
\hline 20 & & PB & 46 & $\mathrm{~F}$ & 46.01 & $46, X X[20]$ \\
\hline 21 & & PB & 67 & $\mathrm{~F}$ & 4.46 & $\begin{array}{l}\text { 46,XX,t(15;17)(q24;q21)[11]/46,idem,inv(6)(p11;2q21),r(11) } \\
(\mathrm{p} 15 ; \mathrm{q} 25)[4] / 46, \text { idem,r(6)(p24;q13)[3]/46,XX[2] }\end{array}$ \\
\hline 22 & & PB & 40 & M & 345.96 & $\begin{array}{l}46, \mathrm{XY}, \mathrm{t}(9 ; 22)(\mathrm{q} 34 ; \mathrm{q} 11.2)[3] / 46, \mathrm{sl}, \mathrm{t}(1 ; 3)(\mathrm{p} 22 ; \mathrm{q} 27)[11] / 47, \mathrm{sdl} 1, \\
\quad+\operatorname{der}(22) \mathrm{t}(9 ; 22)[2] / 53, \mathrm{sdl} 2,+4,+8,+10,+21[\mathrm{cp} 4]\end{array}$ \\
\hline 23 & & PB & 48 & $\mathrm{~F}$ & 137.18 & $46, X X, \operatorname{del}(6)(q 13 ; q 16)[4] / 46, X X[16]$ \\
\hline
\end{tabular}

$\mu 1$ of Hanks' Balanced Salt Solution (HBSS; Welgene, Gyeongsangbuk-do, South Korea) and 1\% FBS (Gibco), and incubated with the antibodies. After washing, the cells were collected using a FACSCanto II flow cytometer (BD Biosciences), and the data were analyzed using FlowJo software (v10; Tree Star Inc., Ashland, OR, USA). PE-labelled mouse anti-mouse NK-1.1 (553165; BD Biosciences), biotin-labelled VEGFR-3 monoclonal antibody (AFL4) (13-5988-82; Invitrogen, Carlsbad, CA, USA), and APC-labelled IFN- $\gamma$ monoclonal antibody (XMG1.2) (17-7311-82; Invitrogen), PE Mouse IgG2a, $\kappa$ Isotype Control (553457; BD Biosciences), Rat IgG2a kappa Isotype Control (eBR2a), Biotin (13-4321-82; Invitrogen), Rat IgG1 kappa Isotype Control (eBRG1), APC (17-4301-82; Invitrogen) were used. Phorbol myristate acetate (PMA; P1585; $50 \mathrm{ng} / \mathrm{ml}$; Sigma), calcium ionophore (A23187, $500 \mathrm{ng} / \mathrm{ml}$; Sigma) and Protein Transport Inhibitor (554724; GolgiStop ${ }^{\mathrm{TM}}$; $2 \mu \mathrm{M}$; BD Biosciences) to measure IFN- $\gamma$ expression in the cells (2 $\mu \mathrm{M}$; BD Biosciences) were used to stimulate the cells for 5 hours. Then, cells were washed with cold fixation and permeabilization solution (554722; BD Biosciences). The biotin signals were detected using streptavidin-APC conjugate (17-4317-82; Invitrogen) as the secondary antibody. The flow cytometry data were analyzed using the appropriate isotype and unstained controls.

\section{Histology}

Spleen, liver, and BM from the euthanized mice were fixed in $4 \%$ paraformaldehyde. BM was fixed in $4 \%$ paraformaldehyde, decalcified with $5 \%$ formic acid and embedded in paraffin. Hematoxylin \& eosin (H \& E) staining was used after fixation to identify leukemia blasts in the tissues, including the fixed spleen, liver, and BM.

\section{Statistical analysis}

All data are presented as the mean \pm standard error (SE). The means were used to compare the data. The results were analyzed by the Kruskal-Wallis test. GraphPad Prism ver. 7 software was used for the analyses. Pannoramic Viewer was used for the analysis of stained cells on the slides. The percentage of stained areas was calculated as the ratio of stained areas to the total area detected in the image. A p-value of $<0.05$ was considered to indicate statistical significance. 


\section{Results}

\section{FLT-4 and IFN- $\gamma$ expressions on AML-NK cells in $\mathrm{BM} / \mathrm{PB}$ in vitro}

$\mathrm{CD} 56^{+} \mathrm{CD}^{-}$lymphocytes are considered NK cells, and AML characterization has been based on multi-parameter analyses including cell morphology, clinical features, immunophenotype, and cytogenetics. To acquire accurate data, only mononuclear cells were gated except blasts and calculated NK cells and IFN- $\gamma$ expression by combinational group both in BM and PB cells (Fig. 2A). Similar with previous data (9), we confirmed that FLT-4 is high on the $\mathrm{CD} 56^{+} \mathrm{CD}^{-}$cells of AML patients and this receptor is relevant to inhibit the release of IFN- $\gamma$ from AML-NK cells, leads to low function and frequency. However, BM-NK cells from AML were increased their frequency, when double and triple combinational treatment, especially MP, AM and AMP group (Fig. 2B). No difference was detected in NK frequency by single treatment. To investigate the expression of IFN- $\gamma$ by treatment, three combinational treatments were applied in ex vivo culture condition. While single treatment has no significant difference in BM cells, MP double group displayed significant increase, compared to other groups. Moreover, the activation of NK cells is almost 3-fold higher than that level of the control group and it shows significant values in MP group compared to the single group (Fig. 2B). In the PB, no group showed a significantly different FLT-4 expression compared to the controls (data not shown). While the frequencies of NK cells were significantly increased in single treatment $(\mathrm{P}, \mathrm{M}$, and $\mathrm{A})$, compared to AML, no significance was detected in dual/triple treated groups in NK cells. Similar with previous data, In IFN- $\gamma$ expression, MP and AMP group showed the significant increase in IFN- $\gamma$ expression, compared to that of AML group (Fig. 2C). The frequency of blasts was also lower in dual and triple treated groups than in AML patients (data not shown). Taken together, our data suggest that designed P may similar efficacy with FLT-4 antagonist in increasing the expression of NK cells and IFN- $\gamma$ in terms of single/double or triple treatments in vitro.
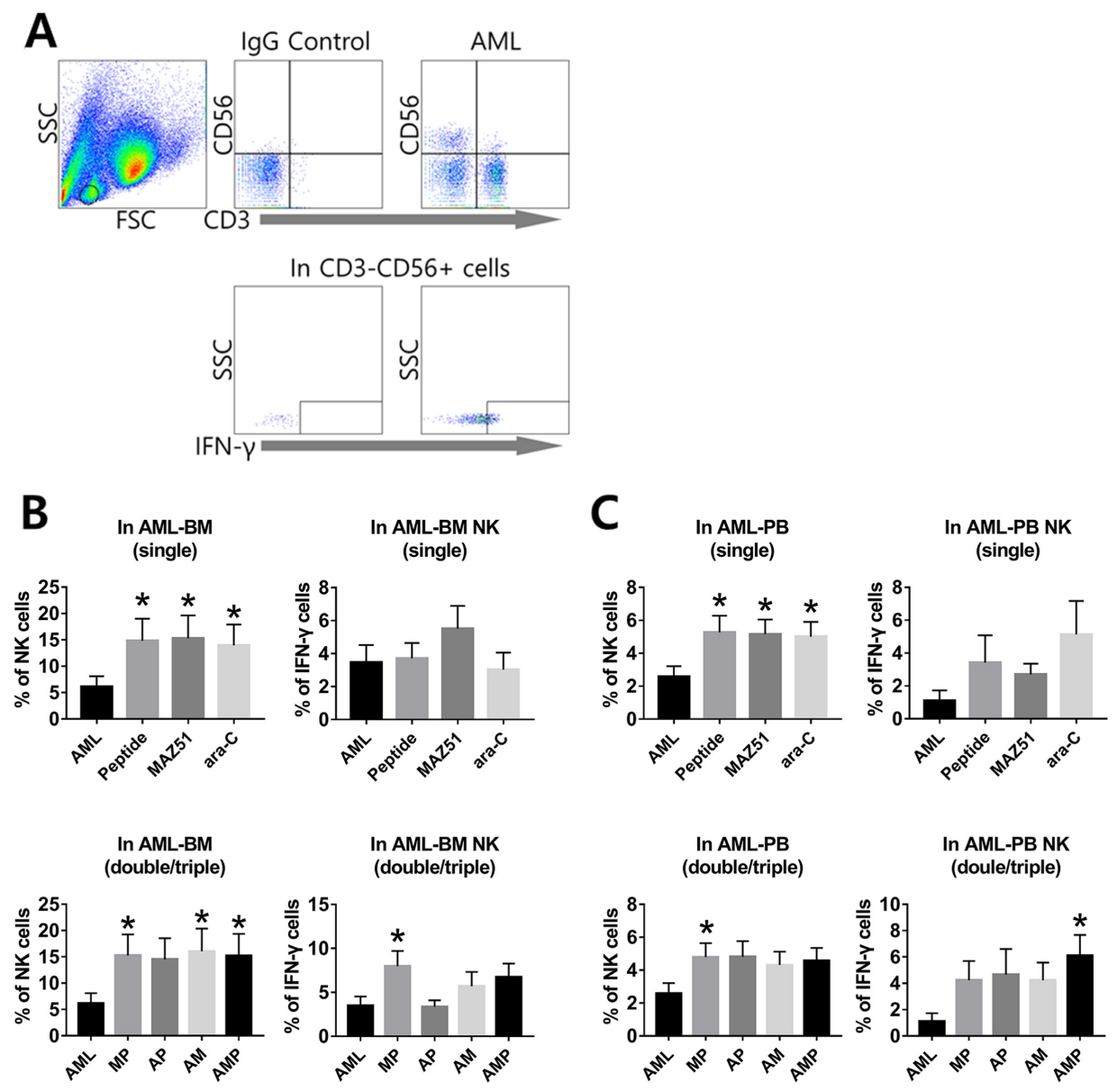

Fig. 2. FLT-4 expressions on AMLNK cells in vitro. (A) $\mathrm{CD}_{56}{ }^{+} \mathrm{CD} 3$ lymphocytes are considered NK cells, and AML characterization has been based on multi-parameter analyses. IFN- $\gamma$ positive NK cells are detected from AML samples. (B) FACS data of single-, double-/triple treatment groups in AML BM. (C) FACS data of single-, double/triple treatment groups in AML PB. Data shown represent the means of independent experiment. Asterisks depict statistically significant differences compared to AML patient $\left({ }^{*} \mathrm{p}\right.$ $<0.05$ vs. AML patients, In BM, 13 AML patients; In PB; 11 AML patients). AML: AML patient, P: peptide, M: MAZ51, A: ara-C. 
The effects of peptide with conventional chemotherapy in the expression of IFN- $\gamma$ from leukemic mice

To address effects of $\mathrm{P}$ with conventional chemotherapeutic agents, a syngeneic leukemic mouse model was developed using C1498 leukemic cells. The protocol shown in Fig. 1 was used in experiments in vivo with tissues including BM, spleen, and liver from each group that were prepared and subjected to FACS analysis. Results in $\mathrm{BM}$ showed that the frequency of NK cells was significantly increased in the $M$ and $A$ single groups and in the AP and AM double groups, compared to leukemic group. No significant difference was detected in the expression of IFN- $\gamma$ (Fig. 3A) and FLT-4 (data not shown). In spleen, the single $M$ and $\mathrm{A}$ treatment groups showed consistently decreased FLT-4 and remarkably increased the NK cells in $\mathrm{P}$ and $\mathrm{M}$ single groups. The frequency of
NK cells in spleen were significantly and synergistically increased by $\mathrm{P}$ regardless of $\mathrm{A}$ exposure, when $\mathrm{M}$ was co-treated; suggesting protection of NK cells by inhibition of FLT-4 (Fig. 3B). The level of IFN- $\gamma$ has no difference, compared to the other groups regardless of combinational types. Meanwhile the frequency of NK cells in liver were protected from apoptosis by $\mathrm{A}$, when $\mathrm{M}$ treated and the expression of IFN- $\gamma$ was significantly increased in AP, $\mathrm{AM}$, and AMP groups, compared to that of leukemic group (Fig. 3C). Also, the transcriptional levels of cytolytic factors, especially IFN- $\gamma$, were highly increased, when dual and triple treatment were applied in AML (Fig. 4). It suggests that designed $P$ partly played the role for protecting the expression of IFN- $\gamma$ in NK cells in tumor microenvironment.
A
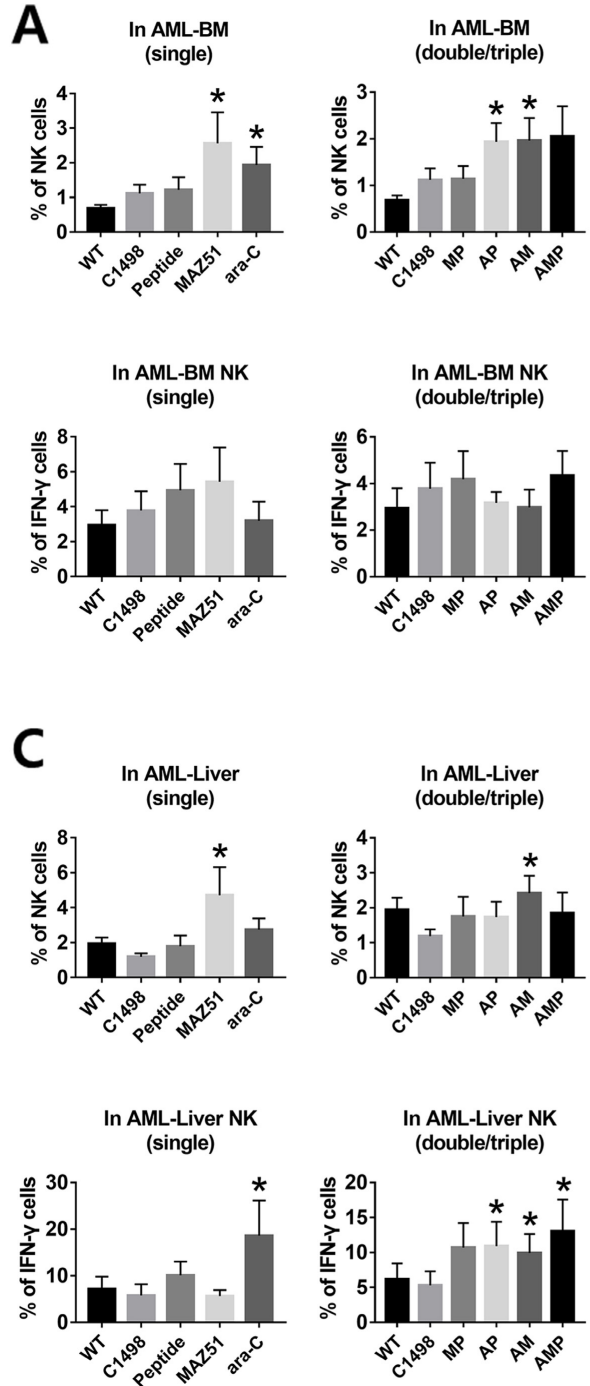
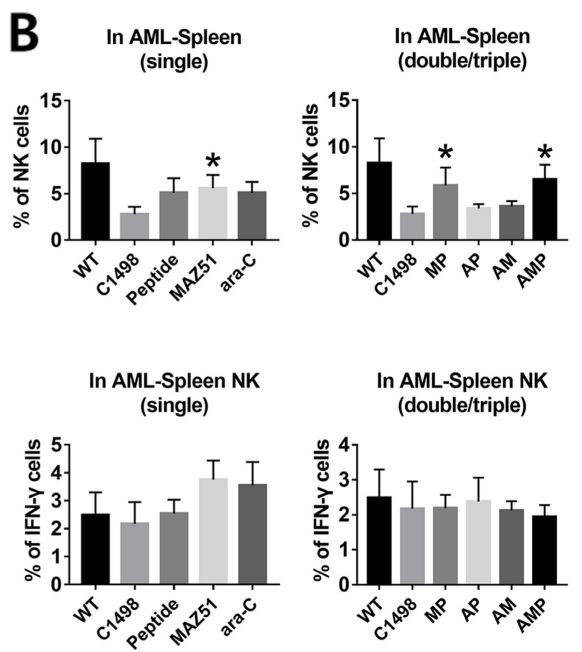

Fig. 3. The effects of peptide targeted FLT-4 in leukemic mouse model. (A) Expressions of NK cells and IFN- $\gamma$ of NK cells in BM after single, double, and triple treatments $(n=12)$. (B) Expressions of NK cells and IFN- $\gamma$ of NK cells in spleen after single, double, and triple treatments $(n=12)$. (C) Expressions of NK cells and IFN- $\gamma$ of NK cells in liver after single, double, and triple treatments. Bars represent an average (mean) \pm SE and Asterisks depict statistically significant differences compared to C1498 injected groups $\left({ }^{*} \mathrm{p}<0.05\right.$ vs. C1498 injected group, $\mathrm{n}=12$ ). WT: wild type, C1498: C1498 injected, P: peptide, M: MAZ51, A: ara-C. 
Spleen LAMP1

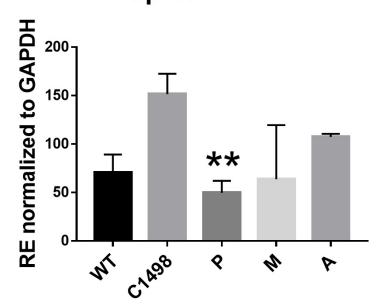

Liver LAMP1

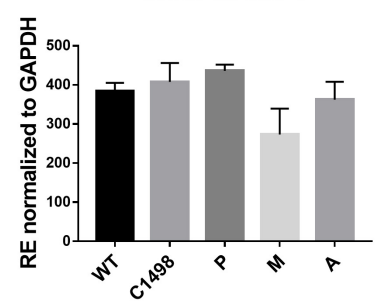

Spleen LAMP1

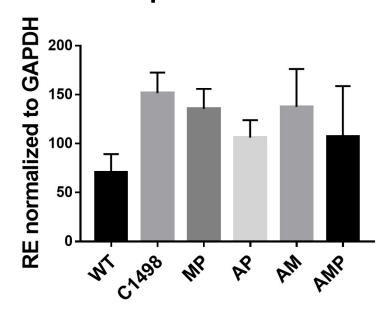

Liver LAMP1

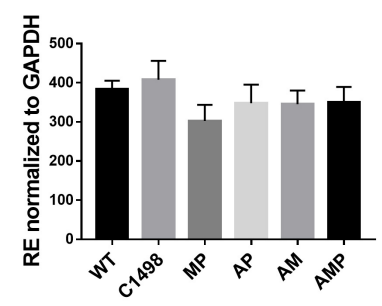

Spleen Perforin

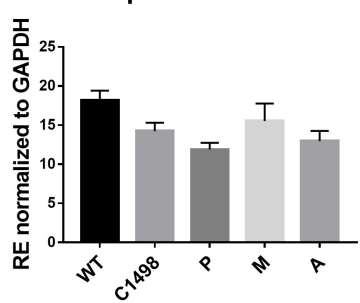

Liver Perforin

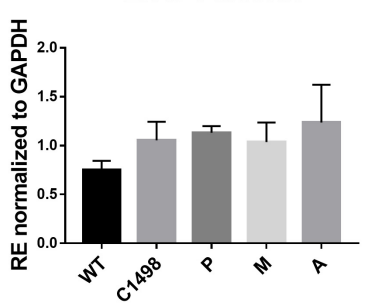

Spleen Perforin

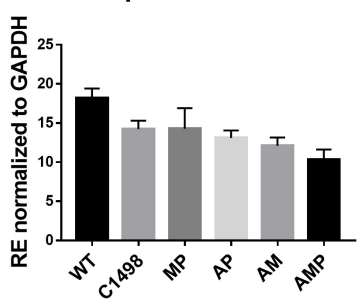

Liver Perforin

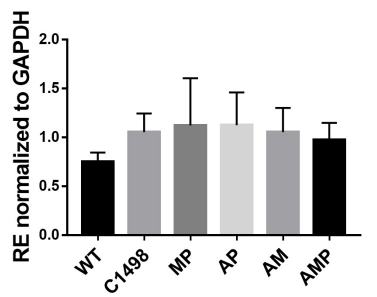

Spleen Granzyme B

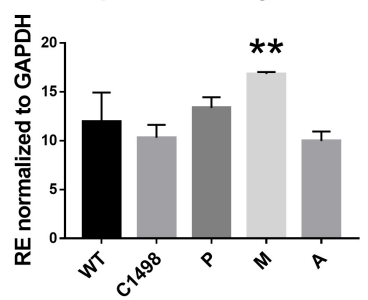

Liver Granzyme B

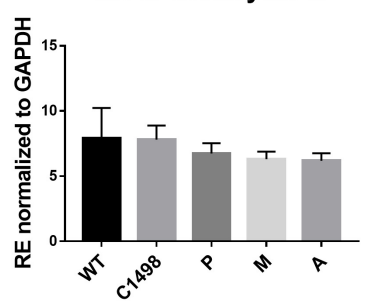

Spleen Granzyme B

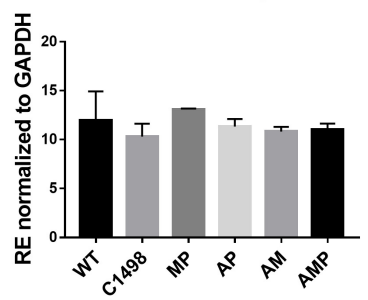

Liver Granzyme B

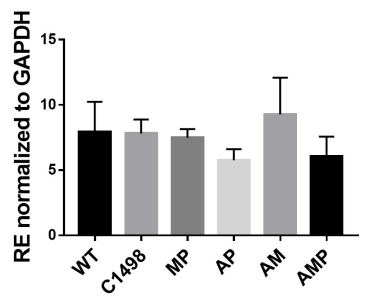

Spleen IFN-y

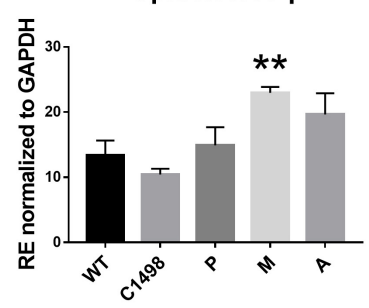

Liver IFN-Y

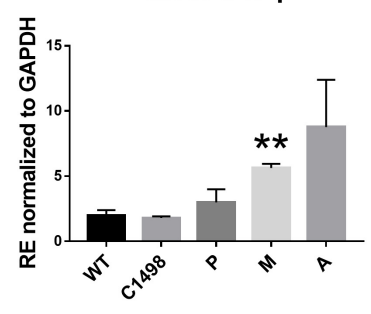

Spleen IFN-Y

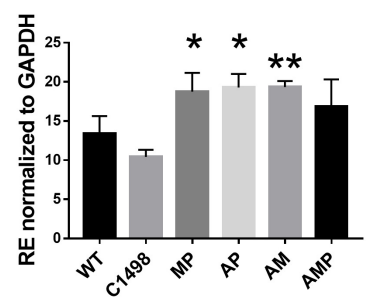

Liver IFN-Y

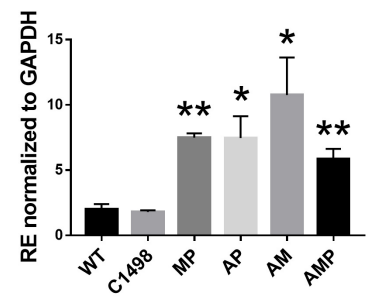

Fig. 4. The effects of peptide targeted FLT-4 in AML mouse model. The transcriptional level of cytolytic factors including LAMP1, perforin, granzyme B, and IFN $\gamma$ displayed in spleen and liver. Bars represent an average (mean) \pm SE and Asterisks depict statistically significant differences compared to $\mathrm{C} 1498$ injected groups. Data shown represent the means of independent experiment with duplicate $\left({ }^{*} p<0.05\right.$ vs. C1498 injected group, $\mathrm{n}=4$ ). WT: wild type, C1498: C1498 injected, P: peptide, M: MAZ51, A: ara-C.

\section{Combinational treatment with peptide and ara-C reduces leukemic blasts in AML mice}

A leukemic model was used to mimic the human leukemia microenvironment. The major organs, such as liver, spleen, and BM, were investigated to confirm the induction of AML mice. When C1498 cells were injected into the $\mathrm{C} 57 \mathrm{BL} / 6 \mathrm{~J}$ mice, the massive infiltration of $\mathrm{AML}$ blasts into diverse tissues was found by H\&E staining, suggesting successful establishment of leukemic mouse model. As shown in Fig. 5A, multiple organ samples from the leukemic mice were examined for pathology to de- termine the effectiveness of each group of single or combination treatments. When we compared the effects of combination treatments, there was a trend toward a reduction in leukemic blasts in the BM, spleen, and liver. The significant suppression of blasts was detected in the A group in vivo condition, compared to other groups in single treatment (Fig. 5B). Interestingly, in the liver, the AM combination showed the best reduction in leukemic blasts compared to the findings in the spleen and BM (Fig. 5). However, double and triple combinations did not consistently reduce the number of leukemic blasts better than 


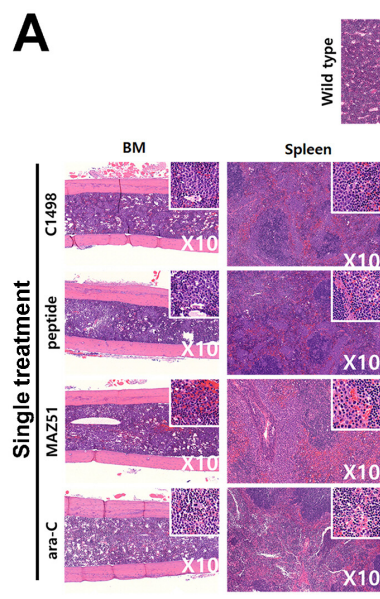

B

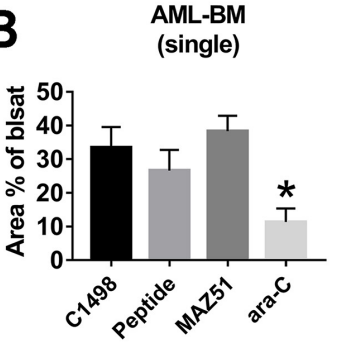

AML-BM (double/triple)

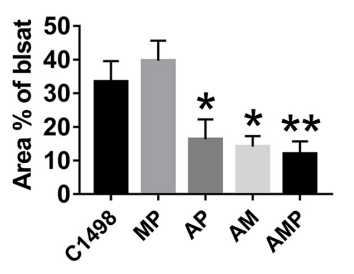

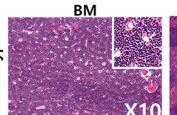
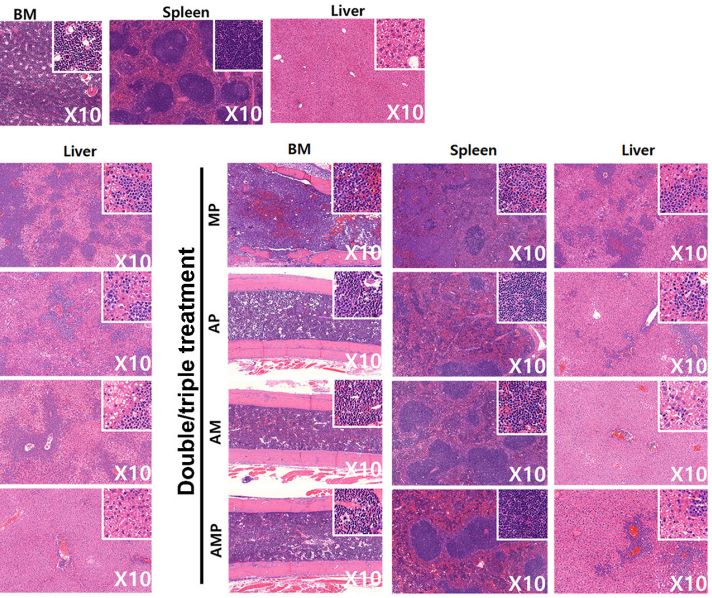

ML-Splee (single)
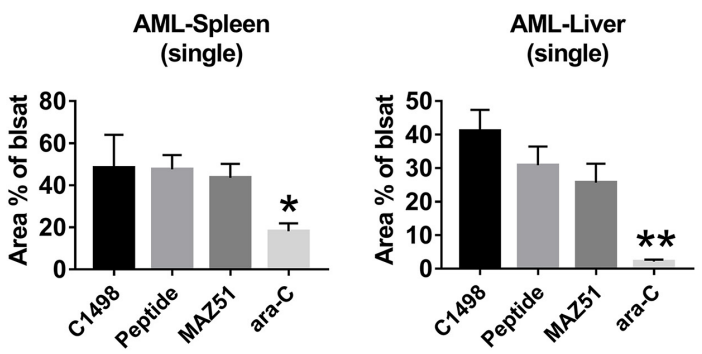

AML-Spleen (double/triple)

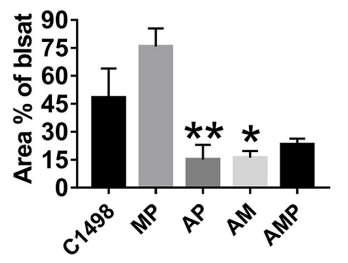

single A treatments. Although P does not fully implement the function of $M$, our results suggest a role for the functional relevance in immune cells to $\mathrm{P}$ that would enable clinical application when combined with $\mathrm{A}$ or similar treatments. Additionally, leukemic mice displayed low survival rates with leukemic properties; however, mice with dual and triple treated groups displayed a long life-span, suggesting therapeutic effect of peptide in AML (Fig. 6).

\section{Discussion}

The aim of this study was originally to reveal whether the higher expression of FLT-4 in AML-NK cells can be downregulated so that the low-killing ability is reversed by some FLT-4 inhibitors. It has been accepted that AML patients have relatively few and low-functioning NK cells and that these cells have poor lytic potential and escape

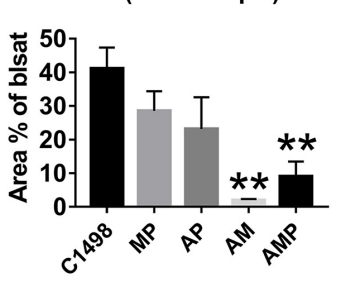
MAZ51, A: ara-C.
Fig. 5. Combinational therapies with peptide and ara-C effectively suppress leukemia blast cells in $\mathrm{AML}$ mice. (A) H\&E staining. BM, spleen and liver from C1498 cells injection mice showed successful engraftment of leukemia cells. Insets (magnification, $\times 40$ ) show enlargement of cells within the main images (magnification, $\times 10$ ). (B) Statistical analysis for the upper panel of a single treated group. And the lower panel for double-, triple treatment groups. Bars represent an average (mean) $\pm \mathrm{SE}$, and asterisks represent statistically significant differences compared to C1498 injected group The indicated values are the averages calculated from at least 5 randomly selected fields of each group of 3 independent experiments $\quad\left({ }^{*} \mathrm{p}<0.05 ; * * \mathrm{p}<0.01\right.$; vs. C1498 injected group, $\mathrm{n}=12$ ). C1498: C1498 injected, P: peptide, M: MAZ51, A: ara-C.

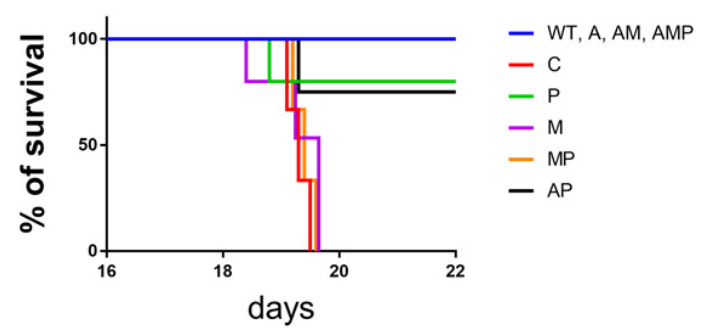

Fig. 6. Comparison of survival over time in the groups. No significant differences in the survival rate were detected between each group, however, dual and triple treated groups displayed a long life span, compared to that of C1498 groups and single only treated groups. It suggests the benefits of peptide and ara- $C$ in $A M L$ treatment. WT: wild type, C1498: C1498 injected, P: peptide, M:

immune surveillance, although some investigators believe there are normal numbers and function at earlier stages of the disease. IFN- $\gamma$ has well-known strong anti-lym- 
phatic and anti-angiogenic effects, causing vessel regression consistently both in murine and human tumors and is considered a main anti-cancer cytokine (17). Previously, our group introduced serial reports of the anti-lymphatic impact of $M$, a chemical against FLT-4, showing the restoration of low-functioning AML-NK cells by inhibiting FLT-4 expression (9). Although the exact action mechanism is still controversial, the positive influence of blocking FLT-4 as a functional activation recovery mechanism in AML-NK cells and the possible modulation of innate immune cells in cancer patients with regard to the lymphatic microenvironmental changes should be further investigated. The findings of single, double, and triple combinational approaches in our experiments partly showed that positively increase in the release of IFN- $\gamma$ either from AML-NK cells or other immune cells with an as yet undefined background by the concurrent results of inhibiting FLT-4 and activating IFN- $\gamma$. Unfortunately, we could not reach a clear conclusion of the impact of our peptide in increasing the function of AML-NK cells in line with the serial results, including a consistent finding of leukemic blast ablation and a reduction in FLT-4 expression in multiple organs of AML mice with markedly increased levels of IFN- $\gamma$ by double or triple combinational treatments, especially compared to the results of single A in this study. Some of the paradoxical organ-specific results in the in vitro and in vivo experiments by single versus double and triple combined agent treatments and inconsistent results in the spleen or liver compared to the BM should be further verified through future studies. However, the present study showed that the newly designed anti-FLT-4 peptide, as in the M experiment results and our previous reports, could restore the low-functioning $\mathrm{CD}_{56}{ }^{+} \mathrm{CD}^{-}$AML-NK cells, which express high levels of the lymphatic marker FLT-4. Our pilot experiments showed an increased expression of lymphatic marker FLT-4 in both AML-NK cells and AML leukemic cells, suggesting the relevance of AML-NK cell activation via the anti-lymphatic cascade. These findings were well-established in our previous reports, which showed a correlation between the induction chemotherapy response and VEGF-C expression in adult AML. The findings further support our thesis that the influx of VEGF-C from the surface of the AML blasts to intracellular migration mechanism in different ways in good vs. bad initial treatment responses should be considered (18). It is not confirmed yet, but the BM may play a critical role in the development of all hematopoietic cells, including NK cells when under the optimal microenvironment for their functional maturation. NK cells need suitable background support from the normal BM and gradually acquire the expression of multiple molecules, such as several adhesion molecules and specific receptors with activating and/or inhibitory functions (19). However, some studies reported that AML-NK cells, which are located in FLT-4-expressing sinusoidal vessels, displayed a much-impaired capacity for releasing IFN- $\gamma$, and the lytic function of perforin/granzyme B in NK cells mainly relied on the murine BM background (20). Based on the findings of enhanced anti-leukemic effects by inducing the immunomodulation of AML-NK cells by blocking with the FLT-4 peptide in this study, the question of how low levels of IFN- $\gamma$ expression in impaired AML-NK cells are associated with lymphatic-prone conditions in both mouse and human AML diseases should be further elucidated. We repeatedly tried to identify a clinically useful anti-FLT-4 peptide by using AML-NK cells with comparatively poor cytolytic activity in the background of AML. According to the literature, many attempts to expand and enhance the number and/or function of AML-NK cells using various cocktails of cytokines and other stimulatory methods to confer increased cytotoxicity of the NK cells in AML patients are ongoing, including our pilot trials revealing the close correlation between FLT-4 and AML-NK cells. Therefore, the positive enhancing effects of the anti-lymphatic $\mathrm{P}$ in the preclinical AML mouse model in this study will be verified further in the next research step. We are not sure how to identify the independent role of anti-FLT-4 peptide on the AML-NK cells or the exact mechanism(s) even in normal NK cells. Consistent with some reports of FLT-4 on multiple tumors, however, AML also strongly expresses lymphatic markers with decreased NK cell function related to the lower release of IFN- $\gamma$, and FLT-4 antagonist peptide restored or improved the functional level of the AML-NK cells. Thus, blocking FLT-4 with a peptide may provide important clues for understanding AML-NK biology and its control by anti-lymphatic peptides. Our preliminary data on the lymphatic microenvironment in AML should be followed by many more scientific experiments, and then we could treat patients with advanced therapeutic modalities to correct unsuitable microenvironments before and/or after chemotherapy and immunotherapy from the beginning of an AML diagnosis. Several FLT-4 inhibitor peptides will be developed in our laboratory or those of others, from which the most efficient peptides should be selected for optimal use in real clinics after well-designed trials.

Taken together, these results suggest that the FLT-4 peptide in combination with conventional chemotherapy may inhibit AML progression more effectively by blocking 
lymphatic markers on AML-NK cells. Although the findings are preliminary, they possibly provide clues and guidance for developing a novel immunotherapeutic strategy for chemotherapy-resistant AML patients in the clinic.

\section{Acknowledgments}

We would like to thank Prof. Gyeongsin Park for comments on the histology data and discussion of this study.

\section{Potential Conflict of Interest}

The authors have no conflicting financial interest.

\section{Funding}

This work was financially supported by a grant from the National Research Foundation (NRF, 2018R1D1A1A0908 355714), Ministry of Education, Republic of Korea.

\section{References}

1. Folkman J. Tumor angiogenesis: therapeutic implications. N Engl J Med 1971;285:1182-1186

2. Kampen KR, Scherpen FJG, Mahmud H, Ter Elst A, Mulder AB, Guryev V, Verhagen HJMP, De Keersmaecker K, Smit L, Kornblau SM, De Bont ESJM. VEGFC antibody therapy drives differentiation of AML. Cancer Res 2018;78:5940-5948

3. de Jonge HJ, Valk PJ, Veeger NJ, ter Elst A, den Boer ML, Cloos J, de Haas V, van den Heuvel-Eibrink MM, Kaspers GJ, Zwaan CM, Kamps WA, Löwenberg B, de Bont ES. High VEGFC expression is associated with unique gene expression profiles and predicts adverse prognosis in pediatric and adult acute myeloid leukemia. Blood 2010;116: $1747-1754$

4. Pizzolo G, Trentin L, Vinante F, Agostini C, Zambello R, Masciarelli M, Feruglio C, Dazzi F, Todeschini G, Chilosi M, Veneri D, Zanotti R, Benedetti F, Perona G, Semenzato G. Natural killer cell function and lymphoid subpopulations in acute non-lymphoblastic leukaemia in complete remission. Br J Cancer 1988;58:368-372

5. Rey J, Fauriat C, Kochbati E, Orlanducci F, Charbonnier A, D'Incan E, Andre P, Romagne F, Barbarat B, Vey N, Olive D. Kinetics of cytotoxic lymphocytes reconstitution after induction chemotherapy in elderly AML patients reveals progressive recovery of normal phenotypic and functional features in NK cells. Front Immunol 2017;8:64

6. Vivier E, Tomasello E, Baratin M, Walzer T, Ugolini S. Functions of natural killer cells. Nat Immunol 2008;9:503-
510

7. Carlsten $M$, Järås $M$. Natural killer cells in myeloid malignancies: immune surveillance, NK cell dysfunction, and pharmacological opportunities to bolster the endogenous NK cells. Front Immunol 2019;10:2357

8. Paul S, Lal G. The molecular mechanism of natural killer cells function and its importance in cancer immunotherapy. Front Immunol 2017;8:1124

9. Lee JY, Park S, Kim DC, Yoon JH, Shin SH, Min WS, Kim HJ. A VEGFR-3 antagonist increases IFN- $\gamma$ expression on low functioning NK cells in acute myeloid leukemia. J Clin Immunol 2013;33:826-837

10. Lee JY, Park S, Min WS, Kim HJ. Restoration of natural killer cell cytotoxicity by VEGFR-3 inhibition in myelogenous leukemia. Cancer Lett 2014;354:281-289

11. Cho HI, Celis E. Optimized peptide vaccines eliciting extensive CD8 T-cell responses with therapeutic antitumor effects. Cancer Res 2009;69:9012-9019

12. Chapel A, Garcia-Beltran WF, Hölzemer A, Ziegler M, Lunemann S, Martrus G, Altfeld M. Peptide-specific engagement of the activating NK cell receptor KIR2DS1. Sci Rep 2017;7:2414

13. Allen TM. Ligand-targeted therapeutics in anticancer therapy. Nat Rev Cancer 2002;2:750-763

14. Borghouts C, Kunz C, Groner B. Current strategies for the development of peptide-based anti-cancer therapeutics. J Pept Sci 2005;11:713-726

15. Stiuso P, Caraglia M, De Rosa G, Giordano A. Bioactive peptides in cancer: therapeutic use and delivery strategies. J Amino Acids 2013;2013:568953

16. Liersch R, Schliemann C, Bieker R, Hintelmann H, Buechner T, Berdel WE, Mesters RM. Expression of VEGF-C and its receptor VEGFR-3 in the bone marrow of patients with acute myeloid leukaemia. Leuk Res 2008; 32:954-961

17. Kak G, Raza M, Tiwari BK. Interferon-gamma (IFN- $\gamma$ ): exploring its implications in infectious diseases. Biomol Concepts 2018;9:64-79

18. Lee SE, Lee JY, Han AR, Hwang HS, Min WS, Kim HJ. Effect of high VEGF-C mRNA expression on achievement of complete remission in adult acute myeloid leukemia. Transl Oncol 2018;11:567-574

19. Abel AM, Yang C, Thakar MS, Malarkannan S. Natural killer cells: development, maturation, and clinical utilization. Front Immunol 2018;9:1869

20. Sciumè G, De Angelis G, Benigni G, Ponzetta A, Morrone S, Santoni A, Bernardini G. CX3CR1 expression defines 2 KLRG1 + mouse NK-cell subsets with distinct functional properties and positioning in the bone marrow. Blood 2011; 117:4467-4475 